\title{
The salivary biomarkers: future clinical investigation technique
}

\section{S. Thamrook*1, Shaiju S Dharan², Madhan Mohan}

Department of Pharmaceutics, Ezhuthachan college of pharmaceutical sciences, Trivandrum, India

\begin{abstract}
Human saliva is a clear, slightly acidic biological fluid containing a mixture of secretions from multiple salivary glands, including the parotid, sublingual gland other minor glands beneath the oral mucosa as well as gingival crevice fluid. Salivary diagnostics has evolved into a sophisticated science and serves as a subset of the larger field of molecular diagnostics, now recognized as a central player in a wide variety of biomedical basic and clinical areas. Saliva biomarkers are source of indicators for local, systemic, and infectious disorders. The saliva based microbial, immunologic, and molecular biomarkers offers unique opportunities to bypass the painful invasive procedures such as biopsies and repeated blood draws by utilizing oral fluids to evaluate the condition of diseased individuals. Accurate and reliable early stage disease detection is the benefit of salivary biomarkers. Salivary biomarkers represent a promising non-invasive approach for oral cancer detection also. This review explains about the salivary biomarkers and their diagnostic approaches.
\end{abstract}

Keywords: Salivary biomarkers; oral cancer; proteomics; metabolomics; transcriptomics.

ISSN: $2581-9143$

Review Article

Corresponding Author

Name: S. Thamrook

Email: thamrushaji9423@gmail.com

\begin{tabular}{l}
\hline \hline Article Info \\
\hline \hline Received on: 06-06-2019 \\
Revised on: 27-07-2019 \\
Accepted on: 10-08-2019 \\
\hline \hline D0I: https://doi.org/10.33974/ijrpst.v1i2.132 \\
\hline \hline \\
Copyright $\odot$ 2019, S. Thamrook, et al., The sali- \\
vary biomarkers: future clinical investigation \\
technique, Production and hosting by Rubatosis \\
Publications. All rights reserved.
\end{tabular}

\section{INTRODUCTION}

Human saliva is composed of $98 \%$ water and $2 \%$ other compounds, such as electrolytes, mucus, antibacterial compounds and various enzymes. Multiple functions of saliva include solubilisation of food substances, food and bacterial clearance, lubrication of soft tissues, bolus formation, swallowing, speech and facilitation of mastication, all of which are related to its fluid characteristics and specific components. Several types of inflammatory biomarkers associated with both oral diseases, as well as systemic diseases have been detected in saliva, such as interleukins, tumour necrosis factor. An increasing number of specific molecular markers for different diseases, such as oral cancer and breast cancer, cardiovascular diseases and human immunodeficiency virus (HIV) and periodontitis are being identified. Oral fluids may contain molecular information capable of communicating an individual's current state of health.[1]

\section{MATERIALS AND METHODS}

Salivary genomics, proteomics, metabolomics an transcriptomics biomarkers: The genomics, transcriptomics and proteomics of saliva represent a non-invasive, safe, and cheap source of complex genetic information (Figure:1). The source of this information complexity is the large variety of DNAs, RNAs and proteins present in the saliva.

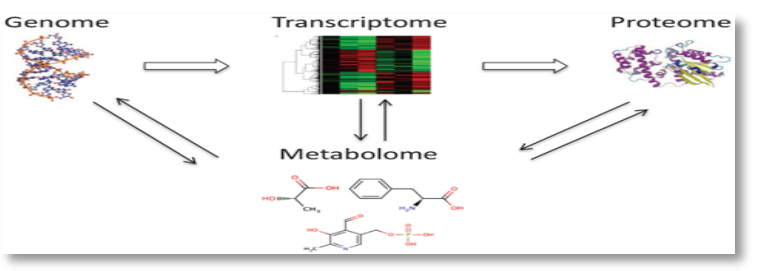

Figure 1: Relation between Salivary genome, transcriptome, proteome and metabolome

Salivary DNAs (genomics) represent the genetic information. Salivary mRNAs provide information on the transcription rates of host genes (transcriptomics) and those of the oral microbes. Other salivary RNAs may indicate RNA-virus 
infections. Salivary proteins (proteomics) represent both genetic information (i.e. the mass- spectrometry reveals amino acid sequences, which help to elucidate the encoding genes) and help to understand the translational regulation of the host body and the oral microbes. Salivary metabolites generates the information about the body diseased condition. ${ }^{[3]}$

\section{Salivary Genome (Human DNA)}

The value of total DNA content in human whole saliva was found in a range between $1.8-128.4 \mu \mathrm{g} / \mathrm{mL}$ with a mean value of $21.6 \mu \mathrm{g} / \mathrm{mL}$. The quality and yield of DNA that can be obtained from saliva is relatively good compared with blood and urine, can be used for genotyping, sequencing, and can be stored without significant degradation. Microarray technology is the method used to detect salivary genome. Salivary DNA is a robust analyte for diagnostics, but is limited to reflecting presence or absence of specific genes, alteration to sequences (mutation) and methylation status, and cannot provide information on up regulation and down regulation of gene expression. ${ }^{[3]}$

\section{Salivary Transcriptome (Human mRNA)}

Although rRNAs and tRNAs are rather stable in the cell, mRNAs are usually rapidly degraded .Because of these reasons mRNA constitutes a small proportion of total cellular RNA comparing to the much more abundant rRNA and tRNA fractions. Consequently, extracellular RNAs belong mostly to rRNA and tRNA fractions, whereas the proportion of mRNA believed to be the smallest. Messenger (m) RNA is the direct precursor of proteins and in general the corresponding levels are correlated in cells and tissue samples. The main strategy to identify salivary transcriptomic biomarkers is through microarray technology. After profiling the transcriptomic biomarkers by microarray, they are validated by quantitative PCR (Polymerase chain reaction), the gold standard for quantification of nucleic acids. Salivary mRNA were localized inside salivary exosomes and these nucleic acids were protected against ribonucleases in saliva. Exosomes are small, right-side out cell-secreted vesicles of about 30-100 $\mathrm{nm}$, derived from fusion of multivesicular bodies to plasma membranes. These regulate the cell-cell environment by altering their gene expression allowing to better understand the molecular basis of oral diseases. ${ }^{[3]}$

Examples: KRAS protein (Kristen rat sarcoma vius), ACRV1 (Acrosomal protein), IL-6 and IL-8.

\section{Salivary Metabolomes}

The metabolome is the complement of small molecule metabolites. Similar to the transcriptome and proteome, the metabolome changes continually and any single profile is a snapshot reflecting gene and protein expression. Metabolomic investigations can generate quantitative data for metabolites in order to elucidate metabolic dynamics related to disease state and drug exposure. Using capillary electrophoresis, these can be identified.[3]

Examples: Tryptophan, Glutamic acid, Phenylalanine, Glutamine and Aspartic acid.

\section{Salivary Proteomes}

The total amount of proteins in whole saliva is ranging between 0.5 to $3 \mathrm{mg} / \mathrm{mL}$. This proteome consists of roughly 1,000 distinct protein sequences, from which around 300 sequences are of human origin. A proportion ranging between $22.8 \%$ to $28.7 \%$ of detected human sequences have an unknown function. Analysis of saliva for protein biomarker discovery has mainly been performed using two dimensional difference gel electrophoresis coupled with MS (which can identify around 300 proteins in a sample), and liquid chromatography based techniques (which can identify more than 1,050 proteins in a sample). Several different classes of salivary protein biomarkers have been reported. Endothelin, a vasoconstrictor, was reported as a potential biomarker for OSCC development.[3]

Examples: AZGP1 (Alpha zinc glycoprotein), Calprolectin etc.

\section{Isolation and screening methods for salivary biomarkers}

Salivary biomarkers isolation and screening are done by different techniques. These include microarray (DNA and RNA), capillary electrophoresis time of flight mass spectrometry, Liquid chromatography/mass spectrometry (LC/MS), matrix-assisted laser desorption ionization-time of flight/mass spectrometry (MALDI-TOF/MS).[4]

\section{PROCEDURE}

\section{Saliva collection}

These are collected by using passive drool.

Passive drool: Passive drool is used for saliva collection (figure: 2). This is mainly used because it is cost effective. To avoid problems with analyte retention or the introduction of contaminants, use only high quality polypropylene vials for collection, such as $2 \mathrm{ml}$ cryovials. The vials used must seal tightly, must be able to withstand temperatures down to $-80^{\circ} \mathrm{C}$, and must be externally threaded to allow for use of the Saliva Collection Aid (SCA) to effectively guide drool directly into the cryovial. A volume of 500 $\mu \mathrm{L}$ whole saliva obtained through the passive drool technique is sufficient to gather DNA for multiple polymorphism assays. [4]

\section{Remove cap from cryovial}

2. Remove SCA from packaging and place securely into cryovial.

3. Instruct participants to allow saliva to pool in the mouth. 
4. With head tilted forward, participants should drool through the SCA to collect saliva in the cryovial.

5. Repeat until sufficient sample is collected. Reserve air space in the vial to accommodate the expansion of saliva during freezing. Collection of samples to be analyzed for multiple analytes may require additional cryovials.

\section{Replace cap onto cryovial.[4]}

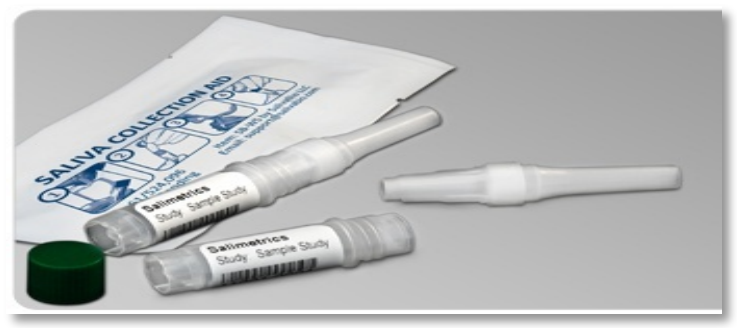

Figure 2: Passive drool

Storage: These can be stored at -80degree Celsius for several years. On the day samples are to be screened, bring samples to room temperature, vortex, and then centrifuge at $1500 \mathrm{x} g$ for 15 minutes. If the samples appear viscous, centrifuge for a greater amount of time, or break up the clot with a pipette tip and recentrifuge. Screening should be performed using only clear saliva.[4]

\section{Isolation and screening methods}

Microarray (DNA and RNA): A DNA microarray is a collection of microscopic DNA spots attached to a solid surface. These used to measure the expression levels of large numbers of genes simultaneously or to genotype multiple regions of a genome. The principle behind microarrays is hybridization between two DNA strands, the property of complementary nucleic acid sequences to specifically pair with each other by forming hydrogen bonds between complementary nucleotide base pairs. A high number of complementary base pairs in a nucleotide sequence means tighter non-covalent bonding between the two strands. After washing off non-specific bonding sequences, only strongly paired strands will remain hybridized. Fluorescently labelled target sequences that bind to a probe sequence generate a signal that depends on the hybridization conditions (such as temperature), and washing after hybridization. Total strength of the signal, from a spot (feature), depends upon the amount of target sample binding to the probes present on that spot. Microarrays use relative quantitation in which the intensity of a feature is compared to the intensity of the same feature under a different condition. RNA also isolated from the cellfree saliva by High-density oligonucleotide microarray. ${ }^{[4]}$

\section{Liquid Chromatography Mass Spectrometry}

Mass spectrometry based proteomics has become the tool of choice for identifying and quantifying the proteome of an organism. The challenges of MS is identify the protein in sample and quantifying their level. In LC-MS-based proteomics, complex mixtures of proteins are first subjected to enzymatic cleavage, then the resulting peptide products are analyzed using a mass spectrometer, which deals with intact proteins and is limited to simple protein mixtures. The experiment has the following key steps (a) extraction of proteins from a sample, (b) fractionation to remove contaminants and proteins that are not of interest, especially high abundance house-keeping proteins that are not usually indicative of the disease being studied, (c) digestion of proteins into peptides, (d) post-digestion separations to obtain a more homogeneous mixture of peptides, and (e) analysis by MS. This technique is highly accurate and sensitive. ${ }^{[4]}$

\section{Capillary Electrophoresis Mass Spectrometry}

This method is mainly used for the detection of metabolites in saliva and quantifying their levels on different conditions. This is an analytical chemistry technique formed by the combination of the liquid separation process of capillary electrophoresis with mass spectrometry. CE-MS combines advantages of both CE and MS to provide high separation efficiency and molecular mass information in a single analysis. It has high resolving power and sensitivity, requires minimal volume and can analyze at high speed. In this technique the metabolite is identified by comparing peak produced with a standard graph. Each metabolite produce different peaks. ${ }^{[4]}$

\section{Matrix Assisted Laser Desorption Ionization (MALDI) TOF MS}

In mass spectrometry this is an ionization technique that uses a laser energy absorbing matrix to create ions from large molecules with minimal fragmentation. It has been applied to the analysis of biomolecules (biopolymers such as DNA, proteins, peptides) and large organic molecules (such as polymers, dendrimers and other macromolecules), which tend to be fragile and fragment when ionized by more conventional ionization methods.

MALDI methodology is a three-step process. First, the sample is mixed with a suitable matrix material and applied to a metal plate. Second, a pulsed laser irradiates the sample, triggering ablation and desorption of the sample and matrix material. Finally, the analyte molecules are ionized by being protonated or deprotonated in the hot plume of ablated gases, and can then be accelerated into whichever mass spectrometer is used to analyse them. This method is highly accurate, but need experts to operate them. By these method the salivary proteins and metabolites can be identified and quantified in different diseased conditions. ${ }^{[4]}$

\section{Salivary biomarkers in oral squamous cell carcinoma}

Oral cancer has emerged as an alarming public health problem with increasing incidence and mortality rates all over the world. The implementation of 
newer screening and early detection approaches are of utmost importance which could reduce the morbidity and mortality associated with this disease. Sensitive biomarkers for oral cancer are likely to be most effective for screening, diagnosis, staging and follow-up for this dreaded malignancy. Unlike other deep cancers, oral cancer is located in oral cavity. Hence, the direct contact between saliva and oral cancer lesion makes the measurement of tumor markers in saliva an attractive alternative to serum and tissue testing. The DNA, RNA and protein molecules derived from the living cancer cells can be conveniently obtained from saliva. Microscopic investigations of the progressive cancer are often conducted too late for intervention. It is also impractical to use imaging techniques for cancer screening, since they are time consuming and expensive. These techniques are typically used for confirmation due to their insensitivity for small lesions. Many biomarkers have been identified from various body fluids. Among which blood and saliva are the most widely studied body fluids that may contain reliable biomarkers for detecting cancer. Saliva has the advantages that it contains low background of normal material and inhibitory substances as well as fewer complexes than blood.[5]

Among all the malignancies, oral cancer is one such malignancy where saliva examination for detection can show the greatest benefit because of its direct contact with oral cancer lesions. Several studies on salivary biomarkers in oral cancer have shown significant clinical usefulness for oral cancer.

Table 1: Clinical significance of salivary biomarkers in OSCC

\begin{tabular}{|c|c|}
\hline Biomarkers & Inference \\
\hline $\begin{array}{l}\text { LDH, MMP- } \\
9\end{array}$ & $\begin{array}{l}\text { All the biomarkers are altered in } \\
\text { oscc }\end{array}$ \\
\hline IL-8, TNF & $\begin{array}{l}\text { These are seen altered in oral can- } \\
\text { cer patients saliva }\end{array}$ \\
\hline Telon & $\begin{array}{l}\text { Increased telomerase activity seen } \\
\text { oral cancer patients }\end{array}$ \\
\hline $\begin{array}{l}\text { P53 autoanti- } \\
\text { bodies }\end{array}$ & $\begin{array}{l}\text { Presence of p53 autoantibodies in } \\
\text { saliva of oral cancer patients }\end{array}$ \\
\hline $\begin{array}{l}\text { Nitrogen spe- } \\
\text { cies }\end{array}$ & $\begin{array}{l}\text { Altered reactive nitrogen species } \\
\text { seen in saliva of oral cancer patients }\end{array}$ \\
\hline IL-6, & $\begin{array}{l}\text { Seen altered in saliva of oral cancer } \\
\text { patients }\end{array}$ \\
\hline
\end{tabular}

Several reports on salivary biomarkers in oral cancer have shown significant clinical usefulness for oral cancer are summarized in (Table 1). As pointed out in the table the exon 4 , codon 63 of the p53 gene was mutated in salivary DNA from five of eight (62.5\%) oral cancers patients. In addition, autoantibodies against p53, the aberrantly expressed protein in patients with oral cancer has been identified in both saliva as well as serum.. It is well established that oxidative stress plays an important role in progression of oral cancer, salivary reactive nitrogen species were significantly higher, while all salivary antioxidants were significantly lower in the oral cancer patients as compared to the controls. This increase in reactive nitrogen species may be the event leading to the consumption and reduction of salivary antioxidants resulting in the oxidative damage to DNA and proteins, and possibly leading to progression of oral cancer. The increased salivary levels of cell cycle regulatory proteins including Cyclin D1 and ki67, glycolytic enzyme lactate dehydrogenase (LDH), matrix metalloproteinase (MMP)-9, as well as reduction in DNA repair enzyme8-oxoquanine DNA glycosylase (OGG1) and Maspin (tumor suppressor protein in oral cancer patients) and significantly increased interleukin (IL)6 levels in saliva of oral cancer patients is identified. The alterations in salivary IL- 6 and tumor necrosis factor alpha (TNF-a) also identified in patients with oral cancer, observed that salivary IL-6 and TNF-a levels were significantly higher as compared to the healthy individuals. IL-6 inactivates p53 tumor suppressor gene by supporting the hypermethylation of its promoter region which results in suppression of apoptosis and uncontrolled cell proliferation. TNFalpha activates NFkB gene(Nuclear Factor kappa B subunit) transcription factor which stimulates cell proliferation and blocks apoptosis and additionally enhances secretion of proinflammatory cytokines. The higher salivary levels of IL-1, IL-6, IL-8 and TNFa in oral cancer patients as compared to the patients with dysplastic oral lesions is also noted. Considering, the fact that same cytokines were significantly elevated in both oral cancer and oral premalignant lesions, it may have a diagnostic value as the marker of malignant transformation of oral premalignant lesions. Telomerase reactivation might be prerequisite for development of malignant cells from the somatic cells by escaping from the proliferative limitations of cellular senescence. These has been directed towards detecting the human papilloma virus (HPV) in saliva, as one of the etiological factor in oral cancer. The incidence of HPV positivity in patients treated for oral cancer is estimated to be more than $45 \%$. With the advances in mass spectrophotometry, there is ongoing development in salivary proteomics for biomarker identification of oral cancer. From depth analysis of human salivary proteomics revealed several salivary proteins at differential levels between oral cancer patients and matched controls. These approach successfully validated five candidate biomarkers including Mac-2 binding protein (M2BP), myeloid related protein 14 (MRP14), CD59, profilin 1 and catalase using immunoassays on independent set of oral cancer patients and matched controls. Using quantitative proteomics approach, also observed consistently increased levels of actin and myosin in saliva samples from individuals with malignant oral lesions as compared to the premalignant lesions. From this concluded that actin and myosin are promising salivary biomarkers for distinguishing premalignant and malignant oral lesions. Salivary transferrin is also 
validated as a biomarker for detection of early stage oral cancer. The tumor-specific DNA in saliva could also be used as biomarker for oral cancer. Methylation array of salivary DNA was supported as an effective biomarker for early detection of oral cancer . Hypermethylation on the promoter of DNA in specific gene such as p16 is found in serum as well as also in saliva. The above mentioned are some of the slivary biomarkers that are significantly used in the early detection of oral cancers. ${ }^{[5]}$

\section{Role of salivary biomarkers in other diseases}

Salivary biomarkers are used in early detection of several diseases as like oral squamous cell carcinoma and various diseases like cardiovascular, renal diseases, pancreatic cancer, perionditis etc. They have also application in some areas like forensic study and physiological research.[6]

\section{Cardiovascular Diseases}

The C-reactive protein (CRP) can be monitored in salivary samples, however CRP remains a nonspecific inflammatory response factor that increases in many disease conditions. Similarly salivary immunoglobulins levels are known to increase in association with coronary artery disease. A group of salivary biomarkers can complement findings of an electrocardiogram (ECG) following an acute myocardial infarction. These markers include CRP, myoglobin and myeloperoxidase, in combination with an ECG, showed a highly significant correlation with myocardial infarct patients as compared to healthy controls. Salivary biomarkers have also been incorporated into POC (Point of care) devices for the rapid assessment of cardiovascular disease (CVD) with potential association with distinct disease stages, demonstrating promising results to identify CVD. Elevated salivary lysozyme levels, a biomarker for oral infection and hyperglycemia, has also shown a significant association with hypertension, an early stage of CVD. In spite of the progress made in biomarker discovery, robust clinical studies are required to validate salivary biomarkers for CVD and its different clinical stages.[6]

\section{Renal Diseases}

Salivary markers are associated with end stage renal disease. The list of markers include cortisol, nitrite, uric acid, chloride, $\mathrm{pH}$, amylase and lactoferrin. The colormetric test strips were used to monitor salivary nitrate and uric acid before and after heamodialysis. It was suggested that a salivary test could be used by patients to decide when dialysis is required, thereby eliminating unnecessary visits to a dialysis clinic. Salivary phosphate has been successfully used as a clinical biomarker for hyperphosphatemia, which is an important contributor to cardiovascular calcification in chronic renal failure (CRF). CRF and heamodialysis patients had significantly higher salivary phosphate levels compared with healthy control subjects. Evaluation of phosphate levels in saliva correlated positively with serum creatinine and the glomerular filtration rate. Salivary phosphate may provide a better marker than serum phosphate for the initiation of treatment of hyperphosphatemia in CRF and HD. These results may also offer new approaches in hyperphosphatemia therapy by establishing measures to bind salivary phosphate in the oral cavity before saliva is swallowed.[6]

\section{Physiological Research}

Stress and pain are often interrelated events. Investigators have attempted to distinguish them using a variety of model systems that induce either stress or pain, and subjects are monitored for changes in salivary biomarkers. Typical markers that have been identified include salivary amylase, cortisol, substance P, lysozyme and secretory IgA. Pain responses in dental pulp have been specifically associated with neuropeptides including calcitonin gene related peptide (CGRP), substance P, neurokinin $A$ and neurokinin P. Salivary testosterone levels have been associated with increased aggressive behavior and also with athletic activities.[6]

\section{Diabetes Mellitus}

Because of the large diabetic population, combined with the current epidemic of Type 2 diabetes, an oral test to monitor blood glucose would be highly desirable. Unfortunately, while it is relatively easy to measure salivary glucose, due to the multiple sources of this material in the oral cavity, salivary glucose levels do not correlate with blood glucose levels. Recent findings demonstrated a proteomic signature in saliva obtained from Type-2 diabetics as compared to control saliva, with 65 proteins showing greater than a 2-fold change. Many of these proteins were associated with metabolic and immune regulatory pathways. These suggest that there may indeed be a unique salivary biomarker profile associated with diabetes. Another approach to detect Type 1 diabetic hyperglycemia involves measuring exhaled methyl nitrate. These demonstrated a correlation between blood glucose levels and exhaled methyl nitrate, due to interaction of superoxide dismutase with nitric oxide as a byproduct of elevated oxidative reactions.[6]

\section{Forensics}

Salivary test have been used for a wide variety of forensic studies. Samples can be obtained from drinking glasses, envelopes, and other sources and then used to detect blood group substances or salivary genetic proteins (primarily proline rich protein polymorphisms). Approximately $85 \%$ of individuals secrete blood-group antigens in their saliva including A, B, H, and Lewis antigens that have been used for identification of individuals in both criminal cases and paternity law suits. With the widespread use of DNA testing, samples of DNA taken from the buccal surface with an oral swab can be easily obtained by untrained individuals. Saliva is 
often present at crime scenes, along with other body fluids, and since DNA is relatively stable in the dry state, these samples can be used to place an individual at the scene of a crime.[6]

\section{Pancreatic Cancer}

Pancreatic cancer is the second most frequent gastrointestinal malignancy. Overall, it is the fourth commonest cause of cancer related mortality, reflecting its advanced stage of presentation. Salivary biomarkers are highly sensitive for the detection of pancreatic cancer. Salivary transcriptome profiles are significantly different between patients with pancreatic cancer and healthy controls. By quantitative PCR technique, the expression levels of two up-regulated mRNAs, KRAS and ACRV1 and three down-regulated mRNAs TK2 (Thymidine kinase), GLTSCR2 (Giloma tumor suppressor candidate region) and CDKL3 (Cyclin dependent kinase like) were significantly different between pancreatic cancer patients with healthy individuals. Further studies find out that combination of four mRNA biomarkers (KRAS, MBD3L2, ACRV1 and DPM1) could differentiate pancreatic cancer patients from non cancer individuals. ${ }^{[6]}$

\section{CONCLUSION}

From this review we understood that the saliva based microbial, immunologic, and molecular biomarkers offers unique opportunities to bypass the painful invasive procedures such as biopsies and repeated blood draws by utilizing oral fluids to evaluate the condition of diseased individuals. Accurate and reliable early stage disease detection is the benefit of salivary biomarkers. Salivary biomarkers represent a promising non-invasive approach for oral cancer detection also. An increasing number of specific molecular markers for different diseases, such as breast cancer, cardiovascular diseases and human immunodeficiency virus (HIV) and periodontitis are being identified. Oral fluids may contain molecular information capable of communicating an individual's current state of health.

\section{REFERENCES}

1. Sharmila DD. (2014). Salivary biomarkers. Journal of pharmaceutical science and research, 5(10), 21012.

2. Shah, F. D., Begum, R., Vajaria, B. N., Patel, K. R., Patel, J. B., Shukla, S. N., \& Patel, P. S. (2011). A review on salivary genomics and proteomics biomarkers in oral cancer. Indian Journal of Clinical Biochemistry, 26(4), 326-334.

3. Zhang, L., Farrell, J. J., Zhou, H., Elashoff, D., Akin, D., Park, N. H., \& Wong, D. T. (2010). Salivary transcriptomic biomarkers for detection of resectable pancreatic cancer. Gastroenterology, 138(3), 949957.
4. Paju, S., Pussinen, P. J., Suominen-Taipale, L., Hyvönen, M., Knuuttila, M., \& Könönen, E. (2009). Detection of multiple pathogenic species in saliva is associated with periodontal infection in adults. Journal of clinical microbiology, 47(1), 235-238.

5. Cheng, Y. S. L., Rees, T., \& Wright, J. (2014). A review of research on salivary biomarkers for oral cancer detection. Clinical and translational medicine, 3(1), 3.

6. Yoshizawa, J. M., Schafer, C. A., Schafer, J. J., Farrell, J. J., Paster, B. J., \& Wong, D. T. (2013). Salivary biomarkers: toward future clinical and diagnostic utilities. Clinical microbiology reviews, 26(4), 781-791.

7. Lee, Y. H., \& Wong, D. T. (2009). Saliva: an emerging biofluid for early detection of diseases. American journal of dentistry, 22(4), 241. 